\title{
Diffusion coefficients for crystal nucleation and growth in deeply undercooled glass-forming liquids
}

\author{
Vladimir M. Fokin \\ S. I. Vavilov State Optical Institute, Ulitsa Babushkina 36-1, 193171 St. Petersburg, Russia \\ Jürn W. P. Schmelzer \\ Institut für Physik, Universität Rostock, Universitätsplatz, 18051 Rostock, Germany \\ Marcio L. F. Nascimento and Edgar D. Zanotto \\ Vitreous Materials Laboratory, Department of Materials Engineering, Federal University of São Carlos, \\ São Carlos, 13595-905 São Carlos, Brazil
}

(Received 14 March 2007; accepted 10 May 2007; published online 19 June 2007)

\begin{abstract}
We calculate, employing the classical theory of nucleation and growth, the effective diffusion coefficients controlling crystal nucleation of nanosize clusters and the subsequent growth of micron-size crystals at very deep undercoolings, below and above $T_{g}$, using experimental nucleation and growth data obtained for stoichiometric $\mathrm{Li}_{2} \mathrm{O} \cdot 2 \mathrm{SiO}_{2}$ and $\mathrm{Na}_{2} \mathrm{O} \cdot 2 \mathrm{CaO} \cdot 3 \mathrm{SiO}_{2}$ glasses. The results show significant differences in the magnitude and temperature dependence of these kinetic coefficients. We explain this difference showing that the composition and/or structure of the nucleating critical clusters deviate from those of the stable crystalline phase. These results for diffusion coefficients corroborate our previous conclusion for the same glasses, based on different experiments, and support the view that, even for the so-called case of stoichiometric (polymorphic) crystallization, the nucleating phase may have a different composition and/or structure as compared to the parent glass and the evolving macroscopic crystalline phase. This finding gives a key to explain the discrepancies between calculated (by classical nucleation theory) and experimentally observed nucleation rates in these systems, in particular, and in deeply undercooled glass-forming liquids, in general. @ 2007 American Institute of Physics. [DOI: 10.1063/1.2746502]
\end{abstract}

\section{INTRODUCTION}

Crystal nucleation and growth determine the overall rate of crystallization. Glass formation on cooling a liquid or on varying any other appropriate thermodynamic parameter is only possible if crystallization - the thermodynamically preferred path of evolution-is inhibited to a sufficient degree. Therefore, a sound knowledge of nucleation and growth processes in glass-forming melts is a key issue to the understanding of vitrification. In addition, advanced glass-ceramic materials-polycrystalline materials produced by controlled nucleation and growth of glasses - can only be manufactured with a desired quality if these two kinetic processes are well understood.

Besides the thermodynamic driving force for crystallization $\Delta G_{V}$ and the specific interfacial energy $\sigma$, the rate of diffusion of the different components of the melt (ions that build the crystalline phase) with an average effective size $a$ through the crystal/liquid interface determines the rates of nucleation and growth of the clusters of the newly evolving crystalline phase. The diffusion rate is determined by an effective diffusion coefficient $D$, which can be estimated from independent experimental determinations of crystal nucleation and growth kinetics.

The value of $D$ can be evaluated via Eq. (1) from the time lag for formation of critical size clusters in nucleation $\tau$,

$$
\tau=\frac{16}{3} \frac{k_{B} T \sigma}{\Delta G_{V}^{2} a^{2} D},
$$

and via Eq. (2) from the rate of kinetically limited (ballistic) growth $U,{ }^{1,2}$

$$
U=f \frac{D}{4 a}\left[1-\exp \left(-\frac{1}{2} \frac{\Delta G_{V} a^{3}}{k_{B} T}\right)\right] .
$$

In both equations, $\Delta G_{V}$ is the thermodynamic driving force per unit volume of crystal, $T$ is the absolute temperature, $a$ is an average (effective) size parameter of the ions building the crystalline phase, and $k_{B}$ is Boltzmann's constant. In Eq. (2), $f$ is a dimensionless parameter describing the different growth modes. For the normal growth mechanism $f=1$, whereas in the case of screw dislocation growth, one has ${ }^{3}$

$$
f \approx \frac{1}{2 \pi} \frac{\left(T_{m}-T\right)}{T_{m}},
$$

where $T_{m}$ is the melting point. For screw dislocation growth at deep undercoolings (corresponding to the maximum of the nucleation rate) $f$ has values of the order of $f \sim 0.1$. To a good approximation, the exponential term in Eq. (2) can be expanded into a Taylor series. Equation (2) can then be written in the form

$$
U=f \frac{D}{8} \frac{\Delta G_{V} a^{2}}{k_{B} T}
$$


TABLE I. Time lag for nucleation and crystal growth rate vs temperature taken from a linear fit of an Arrhenian plot collected from literature data.

\begin{tabular}{cccc}
\hline \hline $\begin{array}{c}\text { Time lag for nucleation } \\
(\mathrm{s})\end{array}$ & $\begin{array}{c}\text { Crystal growth rate } \\
(\mathrm{m} / \mathrm{s})\end{array}$ & $\begin{array}{c}\text { Temperature interval } \\
T(\mathrm{~K})\end{array}$ & References \\
\hline & $\mathrm{Li}_{2} \mathrm{O} \cdot 2 \mathrm{SiO}_{2}$ & & \\
$\exp (-79.185+63602.287 / T)$ & & $693-773$ & $5-10$ \\
& $\exp (31.740-42406.817 / T)$ & $713-783$ & 11 and 12 \\
$\exp (-131.903+120188.79 / T)$ & $\mathrm{Na}_{2} \mathrm{O} \cdot 2 \mathrm{CaO} \cdot 3 \mathrm{SiO}_{2}$ & & 13 and 14 \\
& & $843-877$ & 13 and 14 \\
\hline \hline
\end{tabular}

In Eqs. (1) and (4), it is assumed so far that the thermodynamic and kinetic parameters determining the rates of cluster formation and growth are independent of cluster size. Our own previous analyses and results of other authors show, ${ }^{4}$ however, that these parameters may vary with cluster size and, consequently, may be different for nucleation (nanosize clusters) and growth (micron-size crystals) processes. Taking into account such considerations, we modify Eqs. (1) and (4) and write them in the following more general form:

$$
\begin{aligned}
& \tau=\frac{16}{3} \frac{k_{B} T \sigma}{\Delta G_{V(I)}^{2} a^{2} D_{(I)}}, \\
& U=f \frac{D_{(U)}}{8} \frac{\Delta G_{V(U)} a^{2}}{k_{B} T} .
\end{aligned}
$$

The subscripts $I$ and $U$ assigned to the kinetic and thermodynamic parameters specify the fact that $D$ and $\Delta G_{V}$ may be different for nucleation and growth, respectively.

In this paper we estimate $D$ for crystal nucleation and growth by Eqs. (5) and (6) using independent experimental data on the time lag for homogeneous nucleation and crystal growth rates for two glass-forming liquids: lithium disilicate and sodium calcium metasilicate. Time-lag and crystal growth rates were measured in the same temperature intervals corresponding to very deep undercoolings, which, in the case of lithium disilicate glass, includes the glass transition range.

\section{ESTIMATE OF THE EFFECTIVE DIFFUSION COEFFICIENTS FOR CRYSTALLIZATION OF LITHIUM DISILICATE GLASS IN THE FRAMEWORK OF CNT}

According to the classical nucleation theory (CNT), which is based on Gibbs' description of heterogeneous systems, the thermodynamic driving force for cluster nucleation and growth is considered to be size independent. ${ }^{1,2}$ In other words, the same phase is assumed to determine the state of the critical clusters and of macrocrystals. Therefore, in order to determine the values of the diffusion coefficient following this classical prescription, we employ the thermodynamic driving force $\Delta G_{V(U)}$ for the growth of macrocrystals (the stable lithium disilicate crystal phase) in our computations. Its value can be determined directly by calorimetric methods. Data on nucleation time lag obtained by the "development" method $^{4}$ and crystal growth rates taken from several sources were plotted in Arrhenian coordinates and approximated by a straight line. The results of this procedure were used to estimate the $U(T)$ and $\tau(T)$ shown in Table I. In the case of $\mathrm{Li}_{2} \mathrm{O} \cdot 2 \mathrm{SiO}_{2}$ glass we employed direct experimental values of $U(T)$. Proper thermodynamic data for $\mathrm{Li}_{2} \mathrm{O} \cdot 2 \mathrm{SiO}_{2}$ and $\mathrm{Na}_{2} \mathrm{O} \cdot 2 \mathrm{CaO} \cdot 3 \mathrm{SiO}_{2}$ glasses were taken from Ref. 15. For calculations employing Eq. (1), a value of the specific surface energy equal to $\sigma=0.15 \mathrm{~J} / \mathrm{m}^{2}$ (average value obtained from a previous analysis of experimental nucleation rates with a temperature dependent surface energy ${ }^{15}$ ) was used. One can see from Eq. (1) that the precise value of $\sigma$ does not strongly affect the estimate of $D_{(I)}$. The value of the size parameter $a$ was estimated as $1 \AA$.

Figures 1 and 2 show the effective diffusion coefficients calculated by Eq. (1), $D_{(I)}$, and Eq. (4), $D_{(U)}$, versus temperature in normal and Arrhenian coordinates, respectively. There is a strong difference between $D_{(I)}$ and $D_{(U)}\left(D_{(I)} \ll D_{(U)}\right)$ which reaches about three to four orders of magnitude depending on the assumed growth mechanism. Moreover, $D_{(I)}$ and $D_{(U)}$ have different temperature dependencies corresponding to activation enthalpies of $530 \mathrm{~kJ} / \mathrm{mol}$ for the nucleation time lag and $370 \mathrm{~kJ} / \mathrm{mol}$ for crystal growth. Thus

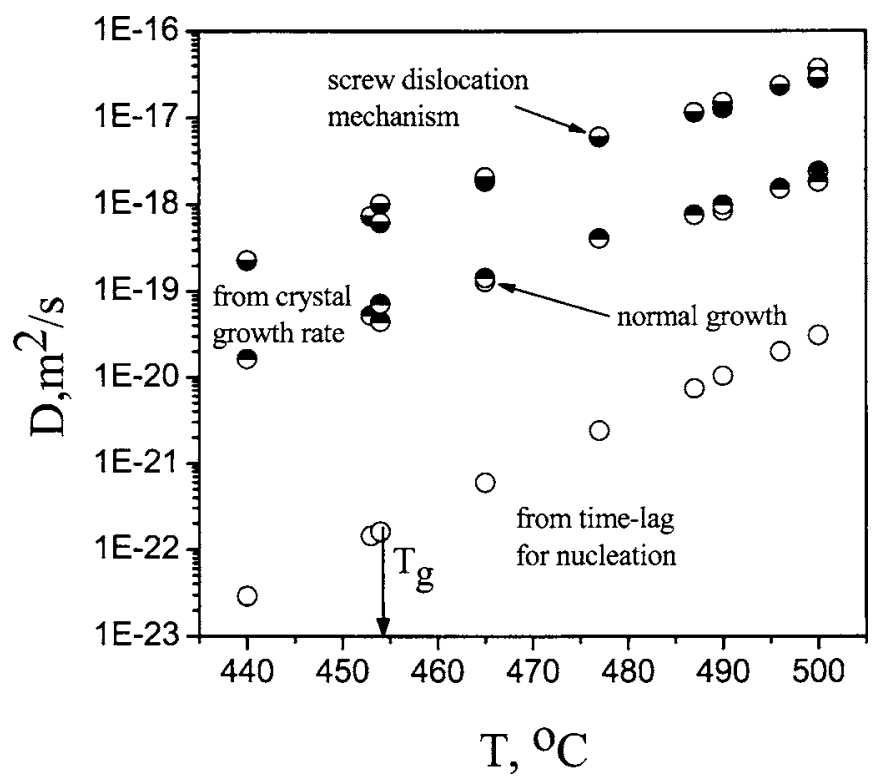

FIG. 1. Diffusion coefficients of $\mathrm{L}_{1} \mathrm{~S}_{2}$ glass estimated from the time lags for nucleation via Eq. (1), and from growth rates for the normal growth and screw dislocation mechanisms via Eq. (4). 


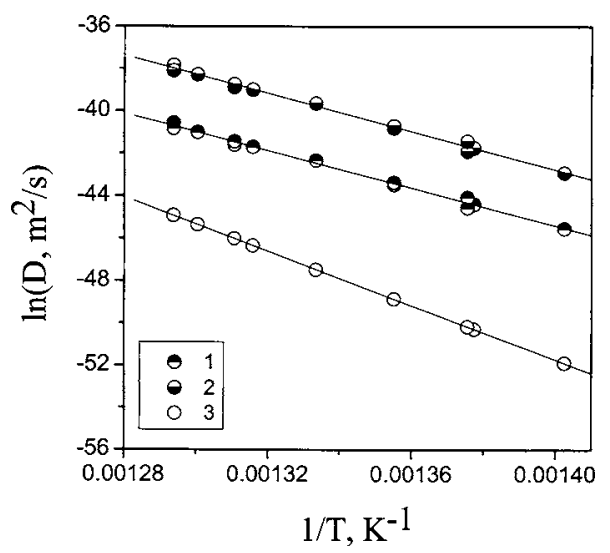

FIG. 2. Arrhenian plot of diffusion coefficients of $\mathrm{L}_{1} \mathrm{~S}_{2}$ glass estimated from the time lags for nucleation (3) via Eq. (1), and from growth rates for the normal (1) and screw dislocation (2) growth mechanisms via Eq. (4).

the use of the main assumptions of CNT resulted in a significant difference between $D_{(I)}$ and $D_{(U)}$. Below we analyze possible reasons for this discrepancy.

\section{ORIGIN OF THE DIFFERENCES IN THE DIFFUSION COEFFICIENTS ESTIMATED FROM NUCLEATION AND GROWTH KINETICS}

In the framework of CNT one expects, in addition to above mentioned thermodynamic requirements, that the nucleation and growth processes are determined by one and the same diffusion coefficient irrespective of cluster size. Such assumption can be considered as a direct consequence of the basic assumption of classical theory that the state of a cluster does not depend on its size. The present result $\left(D_{(I)}\right.$ $\left.\ll D_{(U)}\right)$ thus challenges this main thermodynamic assumption of CNT employed in the derivation of Eqs. (1) and (2). Assuming that the critical clusters and the finally evolving macrophase have similar bulk properties, one should also expect that the effective diffusion coefficients are the same. In contrast to such expectation, however, the difference between $D_{(I)}$ and $D_{(U)}$ reaches several orders of magnitude. According to Eq. (1), it is obvious that the use of a different value of $\sigma$ cannot eliminate this discrepancy. Hence one must look for other factors, which could be responsible for this result. Below we discuss several possible reasons.

It is reasonable to assume that in the case of small (nearcritical) clusters transport of matter from long distances is not required to sustain the aggregation process since a high enough quantity of each of the components is available in the immediate vicinity of the clusters. ${ }^{16}$ The formation of diffusion zones close to such clusters is thus unlikely. This conclusion implies that a kinetically limited (or ballistic) growth can be expected for small clusters independent of their composition. For the system considered, kinetically limited growth takes place also at the macroscopic stage of the phase transformation since the macrocrystals are lithium disilicate and, hence, their composition is the same as that of the parent liquid. Hence the same kinetics could be expected to govern the aggregation process for both small and large clusters. In this way, we come to the conclusion that the nucleating phase (clusters of critical and near-critical sizes) is not lithium disilicate since, otherwise, one should get $D_{(I)}$ $=D_{(U)}$.

It should be recalled that in the early stages of crystallization of this glass at deep undercoolings, metastable phases of $0.1-0.3 \mu \mathrm{m}$ have been detected by transmission electron microscopy ${ }^{17,18}$ simultaneously with the stable $\mathrm{Li}_{2} \mathrm{O} \cdot 2 \mathrm{SiO}_{2}$ $\left(\mathrm{LS}_{2}\right)$ crystals. Metastable phase formation is, however, only one of the possible mechanisms that can explain deviations of the cluster compositions and/or structure for critical and near-critical sized clusters from the parameters reflecting the evolving macroscopic phase. ${ }^{4}$ Thus, at the present stage of analysis, we can conclude with certainty only that the composition and/or structure of the nucleating critical clusters deviate from that of lithium disilicate and, therefore, the thermodynamic driving force for critical cluster nucleation should be smaller than the driving force responsible for macroscopic crystal growth.

Indeed the experimental values of the thermodynamic driving force $\Delta G_{V(U)}$ employed so far in our calculations refer to $\mathrm{LS}_{2}$ macrocrystals. Since $\mathrm{LS}_{2}$ is the thermodynamically most stable phase, the thermodynamic driving force for other thermodynamically possible phases and particularly for the nucleating phase $\left(\Delta G_{V(I)}\right)$ must be smaller than $\left(\Delta G_{V(U)}\right)$. Hence, the following relation holds:

$$
\Delta G_{V(I)}=K \Delta G_{V(U)},
$$

where $0<K<1$. The value of $K$ tends to unity when $T$ $\rightarrow T_{m}$ since at the melting temperature the critical size of the clusters tends to infinity, $r_{*} \rightarrow \infty$, i.e., the critical nucleus becomes a macrophase.

Once the bulk state of the clusters formed in the nucleation stage deviates from the values for the macroscopic phase, the estimate of the diffusion coefficients as performed above must be reconsidered. Since the effective diffusion coefficient depends on the compositions of the clusters and ambient phase, ${ }^{16}$ the difference between $D_{(I)}$ and $D_{(U)}$ could reflect a difference between the compositions of critical clusters and macrocrystals. It is important to emphasize that the real difference between these two types of effective diffusion coefficients would be lower if one assumes that the nucleating phase is not $\mathrm{LS}_{2}$. In order to estimate $D_{(I)}$ via Eq. (1) one must then reduce the thermodynamic driving force for crystallization of the macrophase by the coefficient $K<1$. The introduction of $K$ into Eq. (1) results in an increase of the effective diffusion coefficient estimated from the time lags for nucleation. However, in the general case one cannot calculate the precise value of $D_{(I)}$ since the value of $K$ is unknown. Therefore, one can only claim that $D_{(I)}$ differs from $D_{(U)}$. In particular, a small value of $K$ would considerably reduce the thermodynamic driving force for nucleation, and the inequality $D_{(I)}>D_{(U)}$ could be attained. Such situation could be expected to occur, e.g., if the near-critical clusters are liquidlike, as was proposed earlier in Refs. 1 and 19. 


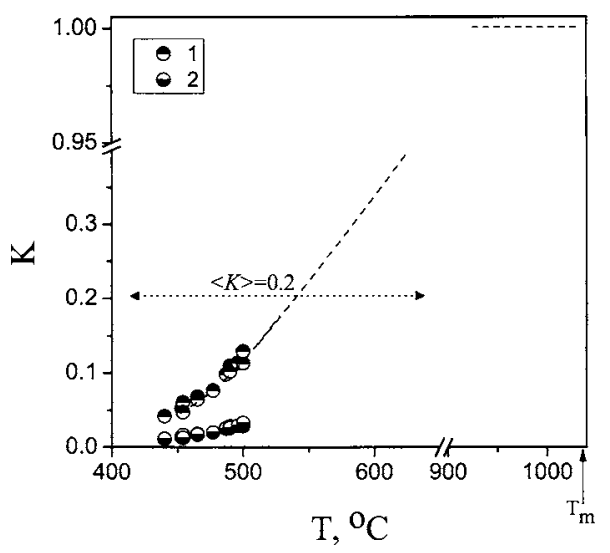

FIG. 3. Coefficient $K$ estimated for $\mathrm{L}_{1} \mathrm{~S}_{2}$ glass for normal growth (1) and screw dislocation growth (2). Dotted line shows the average value of $K$ estimated in Ref. 21 for the temperature interval of $440-680^{\circ} \mathrm{C}$. Dashed lines are placed to guide the eyes.

\section{ESTIMATION OF THE THERMODYNAMIC DRIVING FORCE OF NUCLEATION}

The coefficient $K$ and correspondingly the thermodynamic driving force for nucleation can be estimated only for the special case when the equality $D_{(I)}=D_{(U)}$ is valid. This equality can be assumed to hold approximately if (i) the change of the cluster composition does not affect the effective diffusion coefficient so strongly [see Eq. (3.98) in Ref. 16] or (ii) the critical cluster is a phase of the same composition, e.g., a polymorphic form of $\mathrm{LS}_{2}$. One should recall that situation (ii) occurs in the case of $\mathrm{BaO} \cdot 2 \mathrm{SiO}_{2}\left(\mathrm{BS}_{2}\right)$ glass crystallization, where the high temperature form of $\mathrm{BS}_{2}$ crystals precipitates at low temperatures as the first phase. ${ }^{20}$ This particular case, when

$$
D_{(I)}=D_{(U)}
$$

will be considered below.

Using Eq. (8) together with Eqs. (5) and (6) and replacing $\Delta G_{V(I)}$ by $K \Delta G_{V(U)}$ one can then obtain the following equation for the coefficient $K$ :

$$
K=\left[\frac{2}{3} f \frac{\sigma}{\Delta G_{V(U)} \tau U}\right]^{1 / 2} .
$$

Note that the knowledge of the size parameter $a$ is not required for the determination of $K$. Figure 3 shows the values of $K$ versus temperature estimated via Eq. (9) for normal growth and screw dislocation growth. As we expected, the values of $K$ are smaller than 1, i.e., the thermodynamic driving force for critical cluster formation is smaller than that for $\mathrm{LS}_{2}$ macrocrystals. Hence, as we concluded earlier, the critical nucleus is not the stable lithium disilicate phase.

Another important feature of these results is the increase of $K$ with temperature. Such behavior is quite reasonable since, as we already noted, at $T \sim T_{m}$ the value of $K$ must approach 1. It is clear that the absolute values of $K$ are only estimates being valid at the condition given by Eq. (8). However, it should be recalled that the average value of $K$ for the temperature interval of $440-640{ }^{\circ} \mathrm{C}$ (estimated in Ref. 21 using the effect of dissolution of subcritical nuclei at increasing temperature) was equal to 0.2 . This value is close to that expected from Fig. 3 for normal growth. This result gives indirect evidence for the correctness of the assumption [see Eq. (8)], used for the estimation of the coefficient $K$, even if the composition of the nucleating phase differs from that of the growing macroscopic phase. Thus, we can estimate the thermodynamic driving force for nucleation via Eq. (7) knowing the $\Delta G_{(U)}$ value governing the growth of the stable phase and the coefficient $K$. Consequently, it turns out that they are not the effective diffusion coefficients, but the driving force for crystallization that depends most significantly on the size of the crystallites of the newly evolving phase in the considered system.

However, since the correctness of Eq. (8) for lithium disilicate glass is only an (reasonable) assumption, it is interesting to analyze, in addition, a system for which the composition of critical and near-critical clusters is equal to that of the growing crystals and, respectively, the condition fixed by Eq. (8) is fulfilled with certainty. Such case is found in the early stage of crystallization of sodium calcium metasilicate glasses with compositions between $\quad \mathrm{Na}_{2} \mathrm{O} \cdot 2 \mathrm{CaO} \cdot 3 \mathrm{SiO}_{2}\left(\mathrm{~N}_{1} \mathrm{C}_{2} \mathrm{~S}_{3}\right)$ and $\mathrm{Na}_{2} \mathrm{O} \cdot 1 \mathrm{CaO} \cdot 2 \mathrm{SiO}_{2}\left(\mathrm{~N}_{1} \mathrm{C}_{1} \mathrm{~S}_{2}\right)$, which belong to the field of solid solutions. ${ }^{22}$ In the next section such analysis for a glass with a composition close to $\mathrm{N}_{1} \mathrm{C}_{2} \mathrm{~S}_{3}$ will be presented.

\section{EFFECTIVE DIFFUSION COEFFICIENTS FOR CRYSTAL NUCLEATION AND GROWTH IN THE $\mathrm{Na}_{2} \mathrm{O} \cdot 2 \mathrm{CaO} \cdot 3 \mathrm{SiO}_{2}$ GLASS}

As we have shown earlier, the formation of $\mathrm{Na}_{2} \mathrm{O} \cdot 2 \mathrm{CaO} \cdot 3 \mathrm{SiO}_{2}$ crystals in a glass of the same composition occurs via the nucleation of a solid solution with a composition strongly enriched in sodium, which differs considerably from the stoichiometric parent glass. ${ }^{23}$ With respect to the problem considered, it is important that the composition of critical and near-critical nuclei remain the same (or only slightly changes) in the initial stage of growth when the first nucleated crystals attain macroscopic sizes. ${ }^{14}$ We measured the crystal growth rate in this time interval at low temperatures close to the glass transition temperature together with the steady-state nucleation rate and time lag for nucleation. The kinetic parameters were estimated by fitting the crystal number density versus time curve by the Collins-Kashchiev equation $^{1}$ that describes non-steady-state nucleation. Since the time lags for nucleation and the growth rates measured in this experiment refer to the same phase with a composition differing from that of the stable $\mathrm{N}_{1} \mathrm{C}_{2} \mathrm{~S}_{3}$ phase, we must reduce the thermodynamic driving force for nucleation and growth by the same coefficient $K$. Here the situation is different from that of the lithium disilicate glass, for which only the thermodynamic driving force for nucleation was reduced, because the growth rate referred to the stable macrophase. By proceeding in such a way, Eqs. (5) and (6) can be rewritten as

$$
\tau=\frac{16}{3} \frac{k_{B} T \sigma}{\Delta G_{V(U)}^{2} K^{2}} \frac{1}{D_{(I)} a^{2}},
$$




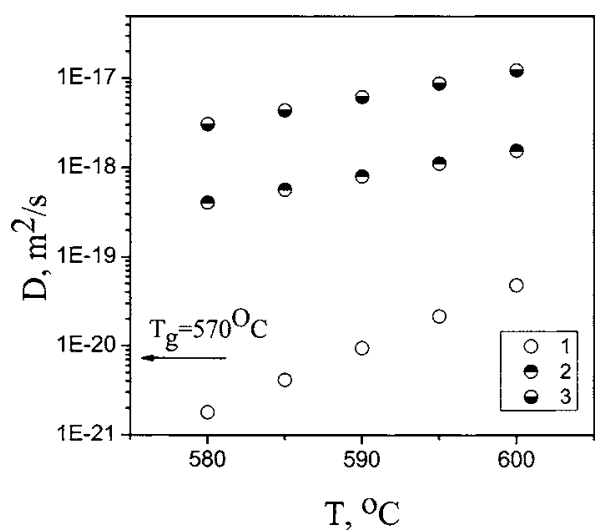

FIG. 4. Effective diffusion coefficients for $\mathrm{N}_{1} \mathrm{C}_{2} \mathrm{~S}_{3}$ glass estimated from time lag for nucleation (1) and crystal growth rates assuming different mechanisms: normal growth (2) and screw dislocation growth (3).

$$
U=\frac{1}{8} f D_{(U)} \frac{\Delta G_{V(U)} K a^{2}}{k_{B} T} .
$$

Figure 4 shows the effective diffusion coefficients estimated from the time lag for nucleation [Eq. (10)] and crystal growth rates [Eq. (11)] - assuming different growth mechanisms - and using the thermodynamic driving force for $\mathrm{Na}_{2} \mathrm{O} \cdot 2 \mathrm{CaO} \cdot 3 \mathrm{SiO}_{2}$ crystals (the most stable phase), i.e., additionally assuming, $K=1$. As for the lithium disilicate glass, $a$ was taken as $1 \AA$ and $\sigma=0.1 \mathrm{~J} / \mathrm{m}^{2}$ was taken from Ref. 15. The resulting values of $D_{(I)}$ are smaller than that for $D_{(U)}$, i.e., the assumption $K=1$ again leads to contradictions. However, as we already noted, the composition of critical nuclei and crystals to which crystal growth measurements refer is the same but different from that of the stable phase $\mathrm{Na}_{2} \mathrm{O} \cdot 2 \mathrm{CaO} \cdot 3 \mathrm{SiO}_{2}$. Hence, the difference in the effective diffusion coefficients obtained in our computations results from the incorrect use of the thermodynamic driving force for $\mathrm{N}_{1} \mathrm{C}_{2} \mathrm{~S}_{3}$ crystals $(K=1)$. Thus we must reduce $\Delta G_{V}$ by a factor $K<1$.

Assuming again that the condition given by Eq. (8) is fulfilled (it holds with good accuracy for the $\mathrm{N}_{1} \mathrm{C}_{2} \mathrm{~S}_{3}$ glass), Eqs. (10) and (11) yield the following formula for the determination of the coefficient $K$ :

$$
K=\frac{2}{3} f \frac{\sigma}{\tau U \Delta G_{V}} .
$$

Figure 5 presents $K$ estimated via Eq. (12) for the normal growth mechanism. As for the lithium disilicate glass, $K$ is less than 1 and increases with increasing temperature. It should be recalled here that the average value of this coefficient in the temperature interval $580-720^{\circ} \mathrm{C}$ estimated from the dissolution effect of subcritical crystals with increasing temperature is $0.13 .^{21}$ Taking into account the increase of $K$ with temperature (see Fig. 5) the values of $K$ obtained in the present study are quite reasonable.

\section{FINAL COMMENTS}

The starting point of the present paper consists of the analysis of the strong difference between the effective diffusion coefficients for nucleation and growth computed based

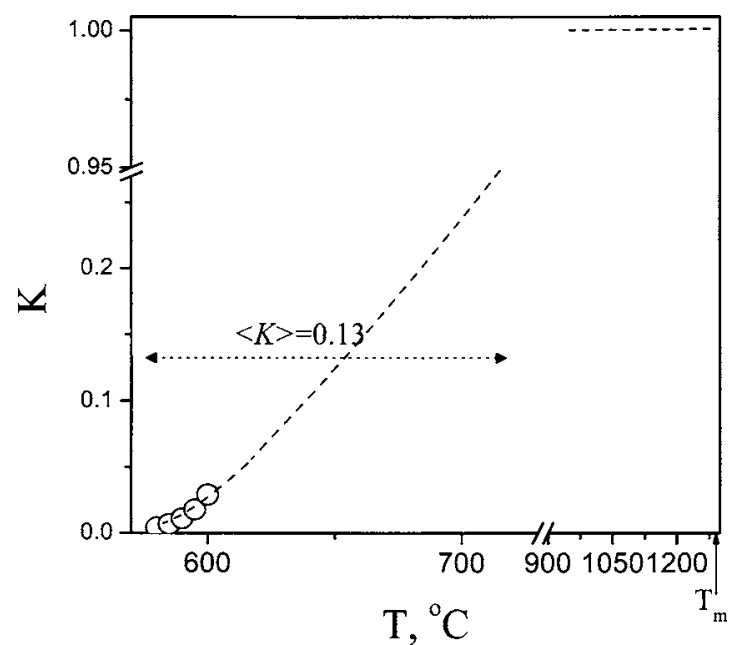

FIG. 5. Coefficient $K$ estimated for $\mathrm{N}_{1} \mathrm{C}_{2} \mathrm{~S}_{3}$ glass assuming normal growth. Dotted line shows the average value of $K$ estimated in Ref. 21 for the temperature interval of $580-720^{\circ} \mathrm{C}$. Dashed lines are placed to guide the eyes.

on experimental data and employing the classical theory of nucleation-growth processes. As previously mentioned, the classical theory employs the assumption that the bulk properties of the clusters are independent of their sizes. The subsequent analysis showed, however, that the real situation is the opposite one. Differences in the diffusion coefficients are found for the considered systems only if the mentioned basic assumption of the classical theory of nucleation and growth is employed in the computations. If this assumption of constancy of bulk cluster properties is released, the experimental data demonstrate a completely different feature. For the considered systems the effective diffusion coefficients are only slightly size dependent, but the state of the clusters is, in general, different from the state of the newly evolving cluster phase and size dependent. Note, however, that in the general case both thermodynamic and kinetic parameters of the clusters of the new phase may be size dependent. ${ }^{24}$

The idea to employ kinetic coefficients estimated from nucleation data to evaluate the nucleation-growth kinetics and to perform computer simulations was proposed some decades ago and remains actual (see, e.g., Refs. 25-29). This approach is based on the assumption that the properties of the newly evolving phase are size independent. However, the crystal growth rates estimated in this way often differ by several orders of magnitude from those measured experimentally. The results of the present paper assuming distinct thermodynamic properties of nanosized critical clusters and macrocrystals could help one to understand such differences.

\section{CONCLUSIONS}

We found significant differences between the effective diffusion coefficients for nucleation and growth computed by the classical theory of nucleation-growth employing experimental data for nucleation time lag and crystal growth rates. The present analysis corroborates our previous findings based on other types of experiments and analysis for the same two glasses. They strongly support the point of view that, even for the so-called polymorphic crystallization, the 
nucleating phase may have a different composition and/or structure compared to the parent glass and the stable macrophase. Such treatment of the crystallization pathway gives a key to explain the often observed discrepancies between calculated via CNT and experimental nucleation rates in deeply undercooled glass-forming liquids.

\section{ACKNOWLEDGMENTS}

The authors express their appreciation for the funding provided by the Brazilian agency FAPESP and by the Deutsche Forschungsgemeinschaft (DFG) for a travel grant to Brazil.

${ }^{1}$ I. Gutzow and J. Schmelzer, The Vitreous State: Thermodynamics, Structure, Rheology, and Crystallization (Springer, Berlin, 1995).

${ }^{2} \mathrm{~J}$. W. Christian, The Theory of Transformations in Metals and Alloys. Part I (Pergamon, Oxford, 1981).

${ }^{3}$ K. A. Jackson, The Nature of Solid-Liquid Interfaces (Dover, New York, 1958).

${ }^{4}$ V. M. Fokin, E. D. Zanotto, N. S. Yuritsyn, and J. W. P. Schmelzer, J. Non-Cryst. Solids 352, 2681 (2006).

${ }^{5}$ V. M. Fokin, A. M. Kalinina, and V. N. Filipovich, J. Cryst. Growth 52, 115 (1981).

${ }^{6}$ A. M. Kalinina, V. N. Filipovich, V. M. Fokin, and G. A. Sycheva, Proceedings of the XIV International Congress on Glass, New Delhi, 1986 Vol. 1, p. 366

${ }^{7}$ P. F. James, Phys. Chem. Glasses 15, 95 (1974).

${ }^{8}$ E. D. Zanotto and M. L. G. Leite, J. Non-Cryst. Solids 202, 145 (1996).
${ }^{9}$ J. Deubener, R. Brückner, and M. Sternitzke, J. Non-Cryst. Solids 163, 1 (1993).

${ }^{10}$ J. J. Tuzzeo, Ph.D. thesis, Ohio State University, 1976.

${ }^{11}$ V. M. Fokin, Ph.D. thesis, Silicate Chemistry Institute, Leningrad, 1980.

${ }^{12}$ L. L. Burgner and M. C. Weinberg, J. Non-Cryst. Solids 279, 28 (2001).

${ }^{13}$ O. V. Potapov, Ph.D. thesis, Silicate Chemistry Institute, St. Petersburg, 2001.

${ }^{14}$ V. M. Fokin, E. D. Zanotto, N. S. Yuritsin, and J. W. P. Schmelzer (unpublished)

${ }^{15}$ V. M. Fokin and E. D. Zanotto, J. Non-Cryst. Solids 265, 105 (2000).

${ }^{16}$ V. V. Slezov, J. W. P. Schmelzer, and A. S. Abyzov, in Nucleation Theory and Applications, edited by J. W. P. Schmelzer (Wiley-VCH, Berlin, 2005), pp. 39-73.

${ }^{17}$ J. Deubener, R. Brückner, and M. Sternitzke, J. Non-Cryst. Solids 163, 1 (1993).

${ }^{18}$ P. C. Soares, Jr., E. D. Zanotto, V. M. Fokin, and H. J. Jain, J. Non-Cryst. Solids 331, 217 (2003).

${ }^{19}$ I. Gutzow and S. Toschev, Krist. Tech. 3, 485 (1968).

${ }^{20}$ A. H. Ramsden and P. F. James, J. Mater. Sci. 19, 2894 (1984).

${ }^{21}$ V. M. Fokin, E. D. Zanotto, and J. W. P. Schmelzer, J. Non-Cryst. Solids 278, 24 (2000)

${ }^{22}$ G. K. Moir and F. P. Glasser, Phys. Chem. Glasses 15, 6 (1974).

${ }^{23}$ V. M. Fokin, O. V. Potapov, E. D. Zanotto, F. M. Spianderelo, V. L. Ugolkov, and B. Z. Pevzner, J. Non-Cryst. Solids 331, 240 (2003).

${ }^{24}$ J. W. P. Schmelzer, A. R. Gokhman, and V. M. Fokin, J. Colloid Interface Sci. 272, 109 (2004).

${ }^{25}$ K. F. Kelton, A. L. Greer, and C. V. Thompson, J. Chem. Phys. 79, 6261 (1983).

${ }^{26}$ Z. Kozisek, Cryst. Res. Technol. 23, 1315 (1988).

${ }^{27}$ K. F. Kelton and M. C. Weinberg, J. Non-Cryst. Solids 180, 17 (1994).

${ }^{28}$ L. Granasy and P. F. James, J. Chem. Phys. 113, 9810 (2000).

${ }^{29}$ M. C. Weinberg, W. H. Poisl, and L. Granasy, C. R. Chim. 5, 765 (2002). 